\title{
Quantification of Carbon Nanotubes Taken up by Macrophage Cells Using Optical Absorption Method*
}

\author{
Minfang Zhang, ${ }^{\dagger}$ Mei Yang, and Toshiya Okazaki \\ CNT-Application Research Center, National Institute of Advanced Science and Technology (AIST), \\ 1-1-1 Higashi, Tsukuba, Ibaraki, 305-8565, Japan \\ Masako Yudasaka \\ Faculty of Science \& Technology, Meijo University, \\ 1-501 Shiogamaguchi, Tempaku-ku, Nagoya 468-8502, Japan and \\ Research Institute of Nanomaterials, National Institute of Advanced Science and Technology (AIST), \\ 1-1-1 Higashi, Tsukuba, Ibaraki, 305-8565, Japan
}

(Received 9 January 2018; Accepted 7 March 2018; Published 20 April 2018)

\begin{abstract}
Quantification of the cellular uptake of nanomaterials is crucial for studies of their toxicity and medical applications. However, our knowledge of the behaviors of carbon nanotubes (CNTs) in cells or tissues remains incomplete due to the lack of appropriate methods for quantitative analysis. Here, we present a unique methodology for quantitatively assessing the cellular uptake of CNTs, taking advantage of their absorption of light in the near-infrared region. Measurement of CNT concentration in cell lysates by monitoring absorbance at $750 \mathrm{~nm}$ enabled highly accurate quantification of CNTs accumulated within cells. In a comparative study of eight commercially available CNTs with size ranging from $30 \mathrm{~nm}$ to $400 \mathrm{~nm}$, we obtained the first quantitative evidence that cellular uptake of CNTs by RAW264.7 macrophages depends on the sizes, specifically on the widths of their bundles in dispersion, regardless of type or manufacturer. [DOI: 10.1380/ejssnt.2018.93]
\end{abstract}

Keywords: Size-dependence; Cellular uptake; Carbon nanomaterials; Quantitive analysis

\section{INTRODUCTION}

With the wide application of carbon nanotubes (CNTs) [1-3] in a variety of industries, the safety of CNTs has become a serious concern in both academia and society. Because toxic effects usually originate from the presence of the toxicant inside cells, quantitative studies of cellular uptake of CNTs are crucial for determining their cytotoxicity. To date, the most widely used technique for investigating cellular uptake of CNTs involves the use of labels, such as fluorescent dyes, attached to the surface of CNTs [4-7]. In biological systems, however, these dye labels might detach from CNTs resulting in uncertainty about whether uptake actually occurred. Furthermore, the fluorescence intensity is quenched over time, preventing confident quantitative interpretation of such assays. These problems could both be overcome by assays that take advantage of the inherent characteristics of unmodified CNTs. For example, CNTs can be detected in solid- or solution-phase bio-samples, such as tissue sections and dispersions [8, 9], using the Raman spectra. Similarly, the quantitative cellular uptake of single-wall CNTs (SWNTs) can be roughly estimated from confocal Raman images and G-band intensities [10, 11]. However, CNTs inside cells easily accumulate and re-bundle, potentially decreasing their Raman intensities. In addition, cellular components might generate a high background, confounding quantitative analysis, especially when CNT uptake is low. Therefore, quantification of the cellular uptake of CNTs remains a challenge.

\footnotetext{
* This paper was presented at the 11th International Symposium on Atomic Level Characterizations for New Materials and Devices '17, Aqua Kauai Beach Resort, Kauai, Hawaii, USA, December $3-8,2017$.

† Corresponding author: m-zhang@aist.go.jp
}

The concentration of CNTs in various dispersions can be easily determined by measurement of optical absorbance based on the Beer-Lambert law [12]. Specifically, CNTs absorb light in the near-infrared (NIR) region, a range of wavelengths in which components of cells and tissues, such as protein and water, have almost no absorption [13]. Therefore, it is possible to measure the cellular uptake quantity of CNTs with less interference from bio-components by measuring the absorption of cell lysates in the NIR region of the spectrum. Here, we apply this method to quantitatively estimate the amount of CNTs inside macrophages.

To extract a general conclusion regarding the relationship between physicochemical properties on cellular uptake, we carried out comparative studies of eight types of commercially available CNTs containing SWNTs or multi-wall CNTs (MWNTs) produced by different manufacturers. When the CNTs were dispersed in water containing bovine serum albumin (BSA), uptake of CNTs by RAW264.7 macrophages depended strongly on the sizes of CNT particles probably the widths of their bundles in the dispersions; specifically, cellular uptake increased with CNT size. Moreover, cell viability measurements revealed that cytotoxicity increased along with CNT uptake. We believe that the method and results described here will help to clarify the mechanism of CNT toxicity, as well as to establish guidelines for managing the hazards of CNTs and related products.

\section{EXPERIMENTAL SECTION}

\section{A. Preparation of CNT dispersions}

Eight types of CNTs were purchased from different manufacturers (Table I). The CNTs were dispersed in aqueous solution of $10 \mathrm{mg} / \mathrm{mL}$ BSA by treatment for 
TABLE I. Characteristics of the CNTs dispersed in BSA

\begin{tabular}{lcccccccc}
\hline \hline CNTs product name & $\begin{array}{c}\text { EC-P } \\
\text { (SWNTs) }\end{array}$ & $\begin{array}{c}\text { HT } \\
\text { (SWNTs })\end{array}$ & $\begin{array}{c}\text { CoMoCAT } \\
\text { (MWNTs) }\end{array}$ & (MWNTs $)$ & $\begin{array}{c}\text { Cnano } \\
\text { (MWNTs) }\end{array}$ & $\begin{array}{c}\text { JEIO } \\
\text { (MWNTs) }\end{array}$ & NIKISO & VGCF-H \\
(MWTs) & (MWNTs) \\
\hline Abbreviation in this study & CNT1 & CNT2 & CNT3 & CNT4 & CNT5 & CNT6 & CNT7 & CNT8 \\
TEM (ave. length, nm) & 162 & 207 & 440 & 161 & 325 & 214 & 2825 & 2080 \\
Zeta potential (mV) & -28 & -32 & -30 & -31 & -32 & -30 & -32 & -35 \\
DLS (nm) & 40 & 65 & 45 & 42 & 60 & 50 & 203 & 225 \\
DCS (ave., nm) & 30 & 45 & 50 & 41 & 51 & 58 & 273 & 398 \\
\hline \hline
\end{tabular}

$5 \mathrm{~h}$ in an ultrasonic homogenizer (VC-750, Sonics \& Materials) [14]. After sonication, the CNT dispersion was centrifuged at $3000 \mathrm{~g}$ for $1 \mathrm{~h}$ and filtered through a cell strainer with a $40 \mu \mathrm{m}$ nylon mesh (Becton, Dickinson \& Company, Franklin Lakes, NJ, USA). The resultant homogenous dispersion of CNTs was maintained as a stock at $2-4{ }^{\circ} \mathrm{C}$. The concentrations of CNTs in BSA solution were adjusted to $\sim 1 \mathrm{mg} / \mathrm{mL}$.

\section{B. Size measurements of CNTs in BSA dispersion}

The size distributions of CNT/BSA dispersions were estimated using differential centrifugal sedimentation (DCS, DC 24000 UHR, CPS Instruments Inc.). For DCS measurements, $100 \mu \mathrm{L}$ samples of each CNT/BSA dispersion were analyzed. The sucrose gradient was $8-24 \%$, and centrifugation was performed at 22,000 $\mathrm{rpm}$. The DCS size of each CNT/BSA dispersion was obtained by calculation based on a standard sample of PVP $(0.239 \mu \mathrm{m})$. The median value in the obtained DCS size distribution was used as the representative particle size. The densities of CNTs for calculating DCS sizes were $1.5 \mathrm{~g} / \mathrm{mL}, 1.782 \mathrm{~g} / \mathrm{mL}$, and $2.1 \mathrm{~g} / \mathrm{mL}$ for SWNTs (CNT1, CNT2) and MWCNTs (CNT3-CNT8), respectively.

\section{Quantification of cellular uptake of CNTs}

For cell experiments, murine macrophage RAW264.7 cells from the European Collection of Cell Cultures were cultured in RPMI medium containing 10\% fetal bovine serum and $1 \%$ streptomycin/penicillin (Gibco) at $37.0^{\circ} \mathrm{C}$ in a $5 \% \mathrm{CO}_{2}$ atmosphere. For quantitative analysis of intracellular CNTs, about $3 \times 10^{5}$ cells were seeded in 6 -well dishes and incubated for $24 \mathrm{~h}$, after which the medium was replaced with CNT-containing medium $(50 \mu \mathrm{g} / \mathrm{mL})$ in three wells or fresh medium in the other three wells. After further incubation for $24 \mathrm{~h}$ or $48 \mathrm{~h}$, the medium was removed, and the cells were rinsed with PBS to eliminate CNTs not taken up by the cells. The cells were then detached from the culture dishes by addition of $0.25 \%$ Trypsin-EDTA. After cells were counted on an automated cell counter (TC20, Bio-Rad), the cell suspension was centrifuged, and the cell pellets were dispersed with $1 \mathrm{~mL}$ of $1: 1$ mixture of CelLytic M Cell Lysis Reagent (Sigma) and $5 \%$ sodium dodecylbenzenesulfonate. The cell dispersions were then sonicated for 10 min with a horn-type sonicator ( $\sim 300 \mathrm{~W}, \mathrm{VC}-750$, Sonics \& Materials). The optical absorption spectra of the cell lysates were then measured in the wavelength range $600-800 \mathrm{~nm}$ on a UVVis-NIR spectrometer. From the absorbance at $750 \mathrm{~nm}$, the concentration of CNTs in cell lysates was estimated based on the corresponding calibration curves [15].

\section{Cytotoxicity evaluation}

Cytotoxicity of the eight types of CNTs in RAW264.7 cells was determined using the cell proliferation reagent WST-1 (Roche). For the WST-1 assay, cells $\left(3.8 \times 10^{4}\right.$ cells per well) were seeded in 96-well plates. After $24 \mathrm{~h}$, the culture medium was replaced with $100 \mu \mathrm{l}$ of culture medium solution containing CNT/BSA. After incubation for $24 \mathrm{~h}$ or $48 \mathrm{~h}$ at $37^{\circ} \mathrm{C}$, the medium was removed, and the cells were rinsed twice with PBS. Next, $100 \mu \mathrm{l}$ of culture medium containing $10 \%$ (vol/vol) WST-1 reagent was added to each well. The absorbance of WST-1-derived formazan was measured using a microplate reader (Model 680 , Bio-Rad, Japan) at $450 \mathrm{~nm}$, with the reference wavelength set at $620 \mathrm{~nm}$.

\section{RESULTS}

\section{A. Size distributions of eight types of CNTs in BSA dispersion}

The abbreviated names of the eight types of CNTs (obtained from the manufacturers) are provided in Table I. The CNTs used in this study included two types of SWNTs (CNT1 and CNT2) and six types of MWNTs (CNT3, CNT4, CNT5, CNT6, CNT7, and CNT8), all of which have different surface areas, diameters, and Raman intensity ratios $(\mathrm{G} / \mathrm{D})$. The CNTs were homogenously dispersed in BSA solution at a concentration of $\sim 1 \mathrm{mg} / \mathrm{mL}$. Zeta potential measurement results showed that all types of CNT/BSA dispersions had almost the same values, around $-30 \pm 5 \mathrm{mV}$ (Table I), indicating that they had similar surface properties and high dispersion stability.

The size distributions of the CNT/BSA dispersions were investigated using transmission electron microscopy (TEM) along with differential centrifugal sedimentation (DCS). TEM images of CNT/BSA dispersions revealed that all types of CNTs were well dispersed as long or short wires without large aggregates (Fig. 1). To quantify the sizes, we measured the lengths of each type of CNTs in ten independent images. The results (Table I) indicated 


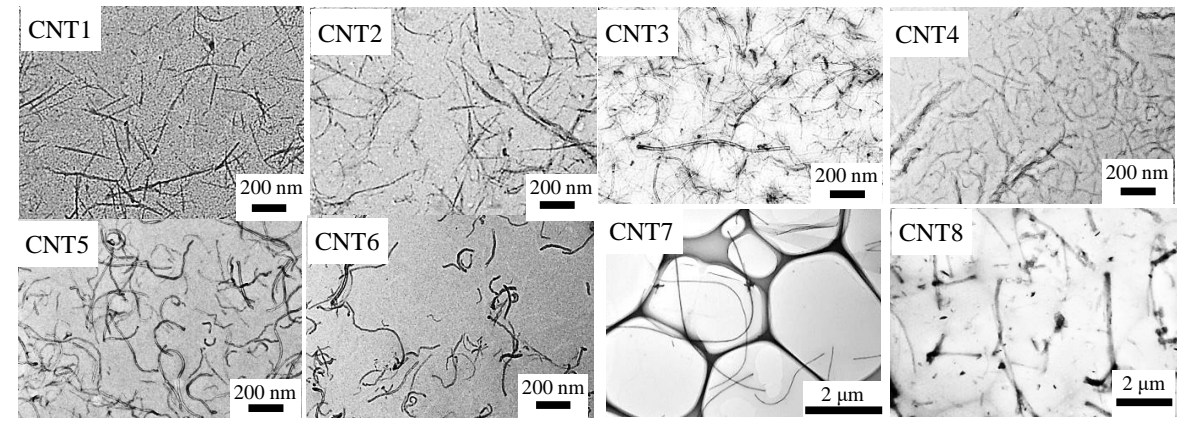

FIG. 1. TEM images of CNTs dispersed in BSA solution. Images were acquired by placing one drop of the CNT dispersions on a TEM grid with carbon film coating.

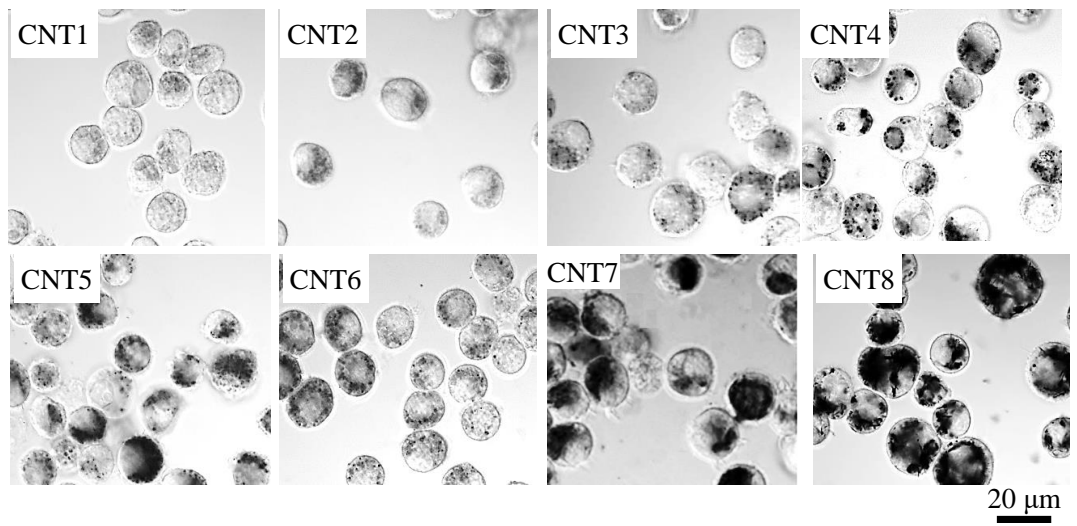

FIG. 2. Confocal microscopy DIC images of RAW264.7 cells incubated with CNTs for 24 h. Black spots represent CNTs. Scale bar, $20 \mu \mathrm{m}$. CNT concentration, $50 \mu \mathrm{g} / \mathrm{mL}$.
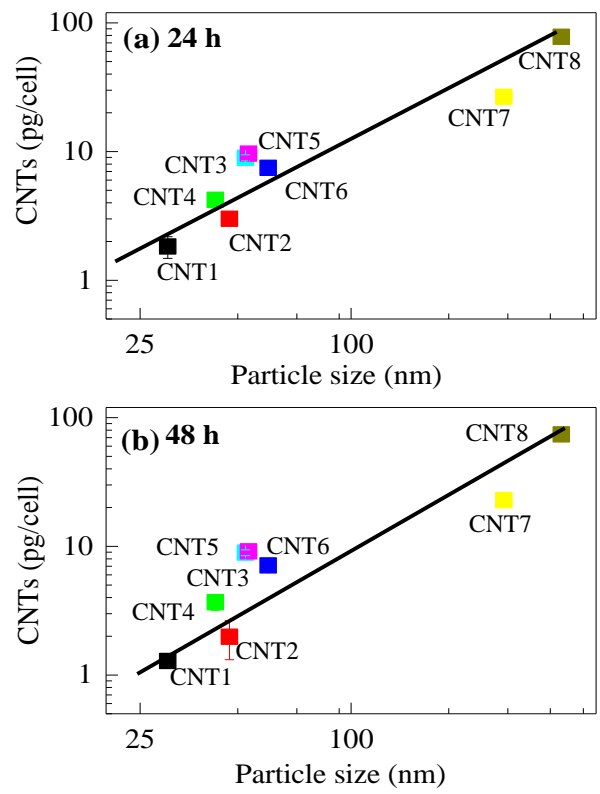

FIG. 3. Quantities of CNTs of different sizes taken up by RAW264.7 cells after incubation for $24 \mathrm{~h}$ (a) or $48 \mathrm{~h}$ (b). The data are expressed as means $\pm \mathrm{SD}$ of three independent replicates. CNT concentration, $50 \mu \mathrm{g} / \mathrm{mL}$.

that CNT8 was the longest (range, 500-4000 nm) whereas CNT1 was the shortest $(\sim 100 \mathrm{~nm})$.

DCS results (Table I) confirmed that CNT8 had the largest particle sizes, with a median around $398 \mathrm{~nm}$, whereas CNT1 was smallest, with a median of $\sim 30 \mathrm{~nm}$.
The size order was as follows: CNT1 $<$ CNT4 $<$ CNT2 $<$ CNT3 < CNT5 < CNT6 < CNT7 < CNT8, consistent with the results obtained by DLS (Table I).

\section{B. Quantification of cellular uptake}

To investigate the size dependence of cellular uptake, we incubated RAW264.7 macrophage cells in medium containing CNT/BSA dispersions, with CNT concentrations of $\sim 50 \mu \mathrm{g} / \mathrm{mL}$. After incubation for $24 \mathrm{~h}$, optical microscopy revealed that the cells were stained to different degrees by CNT uptake (Fig. 2). The dark areas were largest in CNT8-treated cells, and smallest for CNT1. In order of increasing darkness, based on a naked-eye assessment, the samples were ranked as follows: CNT1 < CNT2, CNT3, CNT4, CNT5, CNT6 < CNT7 < CNT8.

For quantification of the uptake of CNTs by RAW264.7 macrophages, the cells were washed with PBS after incubation for $24 \mathrm{~h}$ or $48 \mathrm{~h}$, and then disrupted by addition of cell-lysis buffer and sonication. Homogenous cell lysates containing CNTs were measured by UV-VisNIR spectroscopy, and optical absorbance at $750 \mathrm{~nm}$ was used to estimate the quantity of CNTs in each cell, based on cell number and a calibration curve of CNT concentration. The results (Fig. 3) revealed that the largest CNTs (CNT8, $\sim 390 \mathrm{~nm}$ ) had the highest cellular uptake ( $\sim 75 \mathrm{pg} /$ cell), whereas the smallest CNTs (CNT1) had the lowest uptake $(\sim 1.5 \mathrm{pg} / \mathrm{cell})$. CNTs of similar sizes ( $50 \mathrm{~nm}$ for CNT3, CNT5, and CNT6) had almost the same cellular uptake $(\sim 2-3 \mathrm{pg} /$ cell $)$. These results indi- 


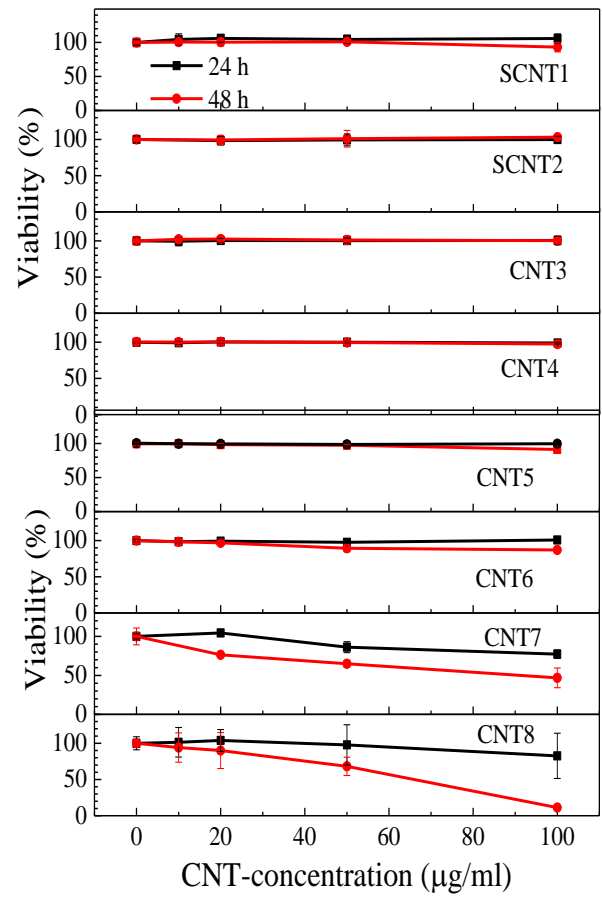

FIG. 4. Cytotoxicity of CNT/BSA in RAW264.7 cells, determined by WST-1 assay at $24 \mathrm{~h}$ (black lines) or $48 \mathrm{~h}$ (red lines). All results are means $\pm \mathrm{SD}$ of six replicated experiments.

cated that the quantity of CNTs taken up by RAW264.7 cells depended on the dispersed particle sizes, but had almost no correlation with the surface area or diameter reported by the manufacturers.

\section{Relation of the cellular uptake of CNTs and cytotoxicity}

The cytotoxicity of CNTs was evaluated using the WST-1 assay, which evaluates mitochondrial activity to monitor cell proliferation and cell death. The results revealed that the viability of RAW264.7 cells did not change remarkably after incubation with CNT1-6 for $24 \mathrm{~h}$ or $48 \mathrm{~h}$, but decreased to $\sim 50 \%$ and $12 \%$ when incubated for $48 \mathrm{~h}$ with a high concentration $(100 \mu \mathrm{g} / \mathrm{mL})$ of CNT7 and CNT8, respectively (Fig. 4). Thus, at a CNT concentration of $100 \mu \mathrm{g} / \mathrm{mL}$, viability was negatively correlated with particle size.

\section{CONCLUSION}

In this study, we developed a method for quantifying the amount of CNTs inside cells, taking advantage of the optical absorption of CNTs in the NIR region. Using this method, we compared the uptake of eight types of commercially available CNTs, including SWNTs and MWNTs, by RAW264.7 macrophages. We found that the cellular uptake of CNTs was dependent on their dynamic particle sizes probably their widths of bundles in test dispersion. Larger CNTs were taken up by macrophages in larger quantities. This result confirmed that the rope-like structures of CNTs have similar properties to those of spherical nanoparticles. This finding will aid in fabrication or design of appropriately sized carbon nanoparticles for various applications, including nanomedicine. In addition, cellular uptake was related to cytotoxicity; specifically, cytotoxicity increased with uptake.
[1] S. Iijima, Nature 354, 56 (1991).

[2] S. Iijima and T. Ichihashi, Nature 363, 603 (1993).

[3] M. F. L. De Volder, S. H. Tawfick, R. H. Baughman, and A. J. Hart, Science 339, 535 (2013).

[4] N. W. S. Kam, Z. Liu, and H. Dai, Angew. Chem. Int. Ed. 45, 577 (2006).

[5] K. Kostarelos, L. Lacerda, G. Pastorin, W. Wu, S. Wieckowski, J. Luangsivilay, S. Godefroy, D. Pantarotto, J. Briand, S. Muller, M. Prato, and A. Bianco, Nat. Nanotechnol. 2, 108 (2007).

[6] B. Kang, J. Li, S. Chang, M. Dai, C. Ren, Y. Dai, and D. Chen, Small 8, 777 (2012).

[7] D. Depan and R. D. K. Misra, Nanoscale 4, 6325 (2012).

[8] Z. Liu, C. Davis, W. Cai, L. He, X. Chen, and H. Dai, Proc. Natl. Acad. Sci. U.S.A. 105, 1410 (2008).
[9] C. G. Salzmann, B. T. T. Chu, G. Tobias, S. A. Llewellyn, and M. L. H. Green, Carbon 45, 907 (2007).

[10] A. Heller, S. Baik, T. E. Eurell, and M. S. Strano, Adv. Mater. 17, 2793 (2005).

[11] B. D. Holt , K. Noel Dahl, and M. F. Islam, Small 7, 2348 (2011).

[12] S. Attal, R. Thiruvengadathan, and O. Regev, Anal. Chem. 78, 8098 (2006).

[13] R. Weissleder, Nat. Biotechnol. 19, 316 (2001).

[14] K. Fujita, M. Fukuda, S. Endoh, H. Kato, J. Maru, A. Nakamura, K. Uchino, N. Shinohara, S. Obara, R. Nagano, M. Horie, S. Kinugasa, H. Hashimoto, and A. Kishimoto, Toxicol. Mech. Methods 23, 598 (2013).

[15] M. Zhang, X. Zhou, S. Iijima, and M. Yudasaka, Small 8, 2524 (2012). 